\title{
Analysis of a Mathematical Model for the Molecular Mechanism of Fate Decision in Mammary Stem Cells
}

\author{
O. U. Kirnasovsky, Y. Kogan, and Z. Agur ${ }^{1}$ \\ Institute for Medical BioMathematics, 10 Hate'ena St., P.O.B. 282, 60991 Bene Ataroth, Israel
}

\begin{abstract}
Recently, adult stem cells have become a focus of intensive biomedical research, but the complex regulation that allows a small population of stem cells to replenish depleted tissues is still unknown. It has been suggested that specific tissue structures delimit the spaces where stem cells undergo unlimited proliferation (stem cell niche). In contrast, mathematical analysis suggests that a feedback control of stem cells on their own proliferation and differentiation (denoted Quorum Sensing) suffices for preserving homeostasis in developing tissues. In the present work we examine this discrepancy by analyzing a mathematical model of signalling pathways in a mammary stem cell. Our work shows that the model has a unique equilibrium depending on the environmental conditions, which is stable for appropriate parameter values. We show how this dependence on the micro-environment can control the cell decision to either proliferate or differentiate. Our work further suggests that this description is sufficient for the creation of niche conditions, with no need of additional structures. The analysis also pinpoints the pathways that may serve as the Quorum Sensing mechanism.
\end{abstract}

Key words: proliferation, differentiation, intracellular pathways, Wnt, Notch, cell signalling AMS subject classification: 92B05, 92C15, 92C37, 37N25

\section{Introduction}

Stem cells (SC) are undifferentiated cells with an unlimited capacity of self-renewal. Differentiation of SCs results in multipotent progenitors, which give rise to lineage-committed progenitor cells from which terminally differentiated cells (DC) originate. In most continuously renewing tissues, SCs divide infrequently, whereas progenitor cells with more limited developmental potential have

\footnotetext{
${ }^{1}$ Corresponding author. E-mail: agur@imbm.org
} 
a large number of rapid divisions [21]. The balance between the unlimited self-renewal capacity of SCs and their ability to continuously supply end cells is regulated by numerous environmental signals acting through paracrine or autocrine pathways [4, 20, 23].

However, even though adult SCs have become a focus of intensive research in the last years [6, $11,18,19,21]$, the complex regulation processes that allow a small population of SCs to replenish depleted tissues (denoted homeostasis), still remain unclear. Such ability requires a sophisticated control on tissue and cellular levels for producing a suitable response to routine demands of tissue renewal and to irregular challenges, such as severe tissue damage. Cell regulation should therefore include some type of feedback, enabling the cell to sense the level of demand in the surrounding tissue and to respond in timely manner. In fact, this control system has to bridge between the intracellular control processes and the extracellular signalling on the tissue level. For maintaining tissue stability the individual SC has to continuously monitor its environment and make its cell fate decisions based on local cues, in addition to global regulation.

In $[1,2,12,13]$ it was suggested and analytically proven that a Quorum Sensing type of control, that is, regulation of proliferation/differentiation balance by a negative feedback of SCs on their own proliferation, suffices for ensuring homeostasis both in normal and in pathological tissues. However, the molecular mechanism underlying this control still remains to be investigated.

In contrast, it has been suggested that SCs require a supporting environment of a defined structure (usually termed "stem cell niche") in order to remain undiffirentiated and proliferate. While such a description represents the common understanding of local SC regulation, no direct experimental data exist for molecularly substantiating such dependence. In the present work we challenge the stem cell niche concept by the alternative, individual cell based, Quorum Sensing concept. Thus, we will mathematically examine the possibility that intracellular processes, which regulate cell cycle and differentiation in mammary SC, also serve as the postulated Quorum Sensing mechanism on the level of the micro-environment.

To this end we analyze a mathematical model for the intracellular and micro-environmental protein interactions in a mammary SC [14], which focuses on the Wnt canonical pathway, the Notch pathway and the E-cadherin pathway, as the drivers of the cellular decision making mechanism. These pathways were selected for their central role in the mammary tissue homeostasis, and also in cancer transformations $[3,5,7,8,9,10,15,16,17,22,24]$. The mathematical model, its biological justification and its implications for cancer progression are presented and discussed in detail in [14]. The aim of the present work is to mathematically analyze this mammary SC mechanistic model in order to understand its fundamental behavior, as influenced by environmental cues.

\section{The Model}

First, we briefly describe the main molecular processes driving fate decisions in a SC of human mammary tissue and the mathematical model describing them. The full model and its biological background are presented and discussed in detail in [14].

The mathematical model is based on the following assumptions about the driving protein in- 
teractions. The decision to proliferate is based on the level of a putative intracellular proliferation factor (PF). If the level of PF reaches a certain threshold, the cell division is initiated irreversibly. Such a factor is a simple representation of several proteins known to promote cell cycle (e.g., cyclin $\mathrm{D})$. The decision to differentiate is controlled by another intracellular factor, termed maturation factor (MF), in a similar way. The regulation processes are modelled as follows: the production of $\mathrm{PF}$ is up-regulated by the intracellular $L E F / T C F$ protein, known to be the main target of Wnt canonical pathway. The production of MF is down-regulated by the intracellular Hes protein. The production rate of Hes protein depends on the activation of Notch receptors by binding of DSL ligands of the neighboring cells. The production of Notch receptors is constant while their degradation is down-regulated by $L E F / T C F$. Thus, $L E F / T C F$ serves as the key protein in this model. Its regulation is performed via Wnt pathway as follows: production of $L E F / T C F$ is increased by Wnt binding the Frizzle receptors, but is down-regulated by $D k k$, which constitutes negative feedback. Importantly, the model assumes that the intracellular Wnt production is constant and therefore is at equilibrium with its degradation. In contrast, the internal production of $D k k$ is assumed to be promoted by $L E F / T C F$. Another important feature of the model is that the regulation of $L E F / T C F$ production depends also on the externally produced Wnt and Dkk. These proteins, secreted by neighboring cells are internalized by the considered cell. An additional regulation pathway is described in the model, which is responsible for cell-cell contacts and growth inhibition, namely, the E-cadherin pathway. The production of E-cadherins is positively regulated by $D k k$ and negatively by $W n t$, while $E$-cadherins themselves contribute to reduction of $L E F / T C F$ synthesis through binding to E-cadherins on the surface of the adjacent cells. The degradation of all the factors (except for the Notch receptor) is assumed to be linear. Non-negative, monotonic, sigmoid shape functions (e.g., Michaelis-Menten or Hill), bounded from above and below and having bounded derivatives, are used to express the influence of some proteins on the production or degradation of other proteins. Since such functions may provide a comprehensive description for several steps in a pathway, the model maintains their general form, as stated in the above properties [14]; see also $[3,5,7,8,9,10,15,16,17,22,24]$.

The above description leads to the following dynamical system, formulated in terms of system of ordinary differential equations [14]:

$$
\begin{aligned}
\dot{P} & =f_{P}^{\uparrow}(L)-\mu_{P} \cdot P, \\
\dot{M} & =f_{M}^{\downarrow}(H)-\mu_{M} \cdot M, \\
\dot{H} & =f_{H}^{\uparrow}(N)-\mu_{H} \cdot H, \\
\dot{N} & =p_{N}-f_{N}^{\downarrow}(L) \cdot N, \\
\dot{L} & =f_{L}^{\downarrow}(E) \cdot f_{W}^{\downarrow}(D)-\mu_{L} \cdot L, \\
\dot{D} & =f_{D}^{\uparrow}(L)-\mu_{D} \cdot D, \\
\dot{E} & =f_{E}^{\downarrow}\left(f_{W}^{\downarrow}(D)\right)-\mu_{E} \cdot E .
\end{aligned}
$$

Here $P$ stands for PF and $M$ stands for MF inside the cell. Other components of the described regulation system are presented by their key proteins: $H$ denotes the amount of Hes-proteins, $N$ -Notch-receptors on the cell surface, $L-L E F / T C F$-proteins, $D-D k k$-proteins secreted by the 
cell, $E$ stands for $E$-cadherins. Additional parameters and functions used to describe the dynamics are:

- $\mu_{P}-$ linear degradation rate for $P$;

- $f_{P}^{\uparrow}(L)$ - production rate of $P$, which is increasing sigmoid in $L$;

- $\mu_{M}$ - linear degradation rate for $M$;

- $f_{M}^{\downarrow}(H)$ - production rate of $M$, which is decreasing sigmoid in $H$;

- $\mu_{H}$ - linear degradation rate for $H$;

- $f_{H}^{\uparrow}(N)$ - production rate of $H$, which is increasing sigmoid in $N$ (later we detail the dependence of this function on the external signal - the amount of DSL receptors on the neighboring cells);

- $p_{N}$ - constant production of Notch receptors;

- $f_{N}^{\downarrow}(L)$ - degradation rate of Notch receptors, which is decreasing sigmoid in $L$;

- $\mu_{L}-$ linear degradation rate for $L$;

- $f_{W}^{\downarrow}(D)$ - production rate of $L$, which is decreasing sigmoid in $D$, this function represents the total signal of the Wnt pathway, including the influence of $D k k$, but not the influence of E-cadherins (later we detail the dependence of this function on the external signals - Wnt and $D k k$ produced by other cells);

- $f_{L}^{\downarrow}(E)$ - the alteration of the production of $L$ due to $E$-cadherin signal (later we detail the dependence of this function on the external signal $-E$-cadherins on the neighboring cells);

- $\mu_{D}$ - linear degradation rate for $D$;

- $f_{D}^{\uparrow}(L)$ - production rate of $D$, which is increasing sigmoid in $L$;

- $\mu_{E}$ - linear degradation rate for $E$;

- $f_{E}^{\downarrow}\left(f_{W}^{\downarrow}(D)\right)$ - production rate of $E$, which is increasing sigmoid in $D$ (the superposition form of this functions stresses that the production of $E$ is based on the same signal of the Wnt pathway, as the production of $L$ ).

The arrows in the functions denotation stand for the direction of the monotonicity. The parameters and the functions are determined by the micro-environment of the considered cell, and by the inner properties of this cell.

In the present analysis we first consider the case of the micro-environmental conditions remaining constant for sufficiently long time, and then explore the change in the behavior of the cell proteins upon alteration in the external signals, e.g., due to division, maturation or apoptosis of another cell in the micro-environment. 


\section{Results}

\subsection{Stability analysis}

The behavior of the proteins $P, M, H$ and $N$ is fully determined by the behavior of the proteins $L, D$, and $E$, due to the equations (2.1), (2.2), (2.3) and (2.4). Therefore for stability studies we first focus on the subsystem of the last three equations.

Examining the three-dimensional subsystem of equations (2.5-2.7) we see that the trajectory with positive initial data will remain in the positive octant. Moreover, this dynamical system is dissipative, because

$$
\dot{D}+\dot{E}+\dot{L}=h(D, E, L)-c \cdot(D+E+L)
$$

for a bounded function $h$ and a constant $c$ (due to boundedness of all the functions used in the system).

We look now for equilibrium points and their stability for the three-dimensional system given by the equations (2.5-2.7). The null-surfaces of the system have the equations

$$
D=\frac{f_{D}^{\uparrow}(L)}{\mu_{D}}, \quad E=\frac{f_{E}^{\downarrow}\left(f_{W}^{\downarrow}(D)\right)}{\mu_{E}}, \quad L=\frac{f_{L}^{\downarrow}(E) \cdot f_{W}^{\downarrow}(D)}{\mu_{L}} .
$$

The conjunction of these three equations is equivalent to the conjunction of the first two of these equations and of the equation

$$
\mu_{L} \cdot L=f_{L}^{\downarrow}\left(\frac{f_{E}^{\downarrow}\left(f_{W}^{\downarrow}\left(\frac{f_{D}^{\uparrow}(L)}{\mu_{D}}\right)\right)}{\mu_{E}}\right) \cdot f_{W}^{\downarrow}\left(\frac{f_{D}^{\uparrow}(L)}{\mu_{D}}\right) .
$$

It is easy to see that the r.h.s. of the equation (3.1) is decreasing in $L$. This implies that the equation has a unique solution, which we denote $L_{S}$. Thus, we have a unique critical point $S=$ $\left(L_{S}, D_{S}, E_{S}\right)$ with $D_{S}=\frac{f_{D}^{\uparrow}\left(L_{S}\right)}{\mu_{D}}, E_{S}=\frac{f_{E}^{\downarrow}\left(f_{W}^{\downarrow}\left(D_{S}\right)\right)}{\mu_{E}}$.

The Jacobi matrix of this system around the unique steady state is

$$
J_{F}(D, E, L)=\left(\begin{array}{ccc}
-\mu_{D} & 0 & \left(f_{D}^{\uparrow}(L)\right)_{L}^{\prime} \\
\left(f_{E}^{\downarrow}\left(f_{W}^{\downarrow}(D)\right)\right)_{D_{\prime}}^{\prime} & -\mu_{E} & 0 \\
f_{L}^{\downarrow}(E) \cdot\left(f_{W}^{\downarrow}(D)\right)_{D}^{\prime} & f_{W}^{\downarrow}(D) \cdot\left(f_{L}^{\downarrow}(E)\right)_{E}^{\prime} & -\mu_{L}
\end{array}\right) .
$$


Then characteristic polynomial is

$$
\begin{aligned}
& \operatorname{det}\left(J_{K}(D, E, L)-\lambda I\right)= \\
& =\operatorname{det}\left(\begin{array}{ccc}
-\mu_{D}-\lambda & 0 & \left(f_{D}^{\uparrow}(L)\right)_{L}^{\prime} \\
\left(f_{E}^{\downarrow}\left(f_{W}^{\downarrow}(D)\right)\right)_{D}^{\prime} & -\mu_{E}-\lambda & 0 \\
f_{L}^{\downarrow}(E) \cdot\left(f_{W}^{\downarrow}(D)\right)_{D}^{\prime} & f_{W}^{\downarrow}(D) \cdot\left(f_{L}^{\downarrow}(E)\right)_{E}^{\prime} & -\mu_{L}-\lambda
\end{array}\right)= \\
& =-\left(\lambda+\mu_{D}\right)\left(\lambda+\mu_{E}\right)\left(\lambda+\mu_{L}\right)+ \\
& +\left(f_{D}^{\uparrow}(L)\right)_{L}^{\prime} \cdot\left(\left(f_{E}^{\downarrow}\left(f_{W}^{\downarrow}(D)\right)\right)_{D}^{\prime} \cdot f_{W}^{\downarrow}(D) \cdot\left(f_{L}^{\downarrow}(E)\right)_{E}^{\prime}+\right. \\
& \left.+\left(\lambda+\mu_{E}\right) \cdot f_{L}^{\downarrow}(E) \cdot\left(f_{W}^{\downarrow}(D)\right)_{D}^{\prime}\right) .
\end{aligned}
$$

By Routh-Hurwitz Theorem, we have (after algebraic transformation) that the criterion for all eigenvalues to have negative real parts is the inequality

$$
\begin{gathered}
f_{W}^{\downarrow}(D) \cdot\left(f_{D}^{\uparrow}(L)\right)_{L}^{\prime} \cdot\left|\left(f_{L}^{\downarrow}(E)\right)_{E}^{\prime}\right| \cdot\left(f_{E}^{\downarrow}\left(f_{W}^{\downarrow}(D)\right)\right)_{D}^{\prime}< \\
<\left(\mu_{D}+\mu_{L}\right)\left(\left(f_{D}^{\uparrow}(L)\right)_{L}^{\prime} \cdot f_{L}^{\downarrow}(E) \cdot\left|\left(f_{W}^{\downarrow}(D)\right)_{D}^{\prime}\right|+\mu_{E}^{2}+\mu_{D} \mu_{L}+\mu_{D} \mu_{E}+\mu_{L} \mu_{E}\right) .
\end{gathered}
$$

This inequality is a sufficient condition of stability of the unique steady state.

The steady-state values of the rest of the variables are uniquely determined by $L_{S}$ :

$$
\begin{array}{ll}
N_{S}=\frac{p_{N}}{f_{N}^{\downarrow}\left(L_{S}\right)}, & H_{S}=\frac{f_{H}^{\uparrow}\left(N_{S}\right)}{\mu_{H}}, \\
M_{S}=\frac{f_{M}^{\downarrow}\left(H_{S}\right)}{\mu_{M}}, & P_{S}=\frac{f_{P}^{\uparrow}\left(L_{S}\right)}{\mu_{P}} .
\end{array}
$$

We can conclude by stating that the entire system (2.1-2.7) has a unique equilibrium point. This point is locally asymptotically stable if the above given condition on the parameters is fulfilled. In particular, one can choose appropriate functions and parameters, to obtain the system with unique stable equilibrium and a large enough basin of attraction, so that from any biologically relevant initial state the system will converge to this equilibrium. Numerical simulations suggest that, using Hill functions, stable behaviour can be observed for a relatively wide range of parameters and initial values - in each one of 10,000 simulaitons with randomly chosen parameteres and initial points, convergence toward the fixed point was observed (data not shown).

\subsection{Rationalizing the system behavior — dependence on external signals}

The full global behavior of the system cannot be determined from the preceding analysis, except for dissipation and positiveness. However, local stability allows one to study some important biological question. 
Suppose the system's initial state is a stable equilibrium and assume further that some of its parameters are determined by the micro-environmental conditions. A change in these conditions is expected to alter the parameter values so that now the system has a new equilibrium point. From the biological point of view, we can assume that the current point is close enough to the new equilibrium so that the system remains in its basin of attraction. Therefore, in a relatively short time the system will evolve towards a new equilibrium point, in particular, attaining new values of $P_{S}$ and $M_{S}$. If, as a result, one of the two variables, $P$ or $M$, reaches its characteristic threshold, the cell is driven into proliferation or differentiation, respectively. This allows to view this system as a logical device switching its states (quiescence, proliferation or maturation) following environmental signals.

Our primary interest is to examine the cell response to changes in its local environment, such as increase or decrease in the number of neighbors. In terms of our model, such events will produce feedbacks realized in some of the signals received by the cell. In our model these signals include (i) the level of external $D k k$ entering into the cell, $D_{e}$; (ii) the level of external Wnt entering the cell, $W_{e}$; (iii) the level of $D S L$ receptors on the neighboring cells, $N_{L}$; and (iv) the level of E-cadherins on the surface of the neighboring cells, which determines the proportion of bound E-cadherins, $k_{E}$. The latter are taken as parameters in the functions in the r.h.s. of our system, so we rewrite our system to explicitly formulate the dependence of the functions on the external signals:

$$
\begin{aligned}
\dot{P} & =f_{P}^{\uparrow}(L)-\mu_{P} \cdot P, \\
\dot{M} & =f_{M}^{\downarrow}(H)-\mu_{M} \cdot M, \\
\dot{H} & =f_{H}^{\uparrow}\left(N \cdot h\left(\frac{N_{L}}{N}\right)\right)-\mu_{H} \cdot H, \\
\dot{N} & =p_{N}-f_{N}^{\downarrow}(L) \cdot N, \\
\dot{L} & =f_{L}^{\downarrow}\left(k_{E} \cdot E\right) \cdot f_{W}^{\downarrow}\left(D_{e}+D, W_{e}\right)-\mu_{L} \cdot L, \\
\dot{D} & =f_{D}^{\uparrow}(L)-\mu_{D} \cdot D, \\
\dot{E} & =f_{E}^{\downarrow}\left(f_{W}^{\downarrow}\left(D_{e}+D, W_{e}\right)\right)-\mu_{E} \cdot E .
\end{aligned}
$$

The functions depend on the external signals as follows:

- $f_{H}^{\uparrow}\left(N \cdot h\left(\frac{N_{L}}{N}\right)\right)-N$ is multiplied by the smooth function $h\left(\frac{N_{L}}{N}\right)$, whose derivative is a smooth approximation of a step function that drops from a positive value to zero at some point (this means that $h(x)$ is nearly linear up to some point, following which it becomes nearly constant; this property reflects the hypotheses that Notch receptors are activated upon binding the ligands and that binding is immediate and total);

- $f_{W}^{\downarrow}\left(D_{e}+D, W_{e}\right)-D_{e}$ is added to $D$, the internal $D k k$; while $f_{W}^{\downarrow}$ increases in $W_{e}$ (recall that we assume that the internal Wnt has a constant intracellular level);

- $f_{L}^{\downarrow}\left(k_{E} \cdot E\right)-k_{E}$ reflects the proportion of binding of cell E-cadherins by the E-cadherins of the adjacent cells, and is directly related to the number of E-cadherin receptors on the neighboring cells, which increases with increase in the number of the adjacent cells. Note 
that from the formal point of view, the function $f_{L}^{\downarrow}$ has been changed with respect to equation (3.8).

To analyze the switch in cell behavior we rewrite the steady-state equation (3.1) of our system, with explicit dependence on parameters, as in (3.2-3.8):

$$
\mu_{L} \cdot L=f_{L}^{\downarrow}\left(k_{E} \cdot \frac{f_{E}^{\downarrow}\left(f_{W}^{\downarrow}\left(W_{e}, D_{e}+\frac{f_{D}^{\uparrow}(L)}{\mu_{D}}\right)\right)}{\mu_{E}}\right) \cdot f_{W}^{\downarrow}\left(W_{e}, D_{e}+\frac{f_{D}^{\uparrow}(L)}{\mu_{D}}\right) .
$$

This allows to determine the possible influence of each of the parameters on the steady state of the system and, in particular, on the values of $L_{S}, M_{S}$ and $P_{S}$. Recall the assumption that the levels of the PF and MF, represented in our system by $P$ and $M$, determine the cell fate: when both are low, the cell remains quiescent; when one of them reaches its critical threshold, the cell irreversibly goes into division or differentiation. Below, we show how alteration of external signal contributes to either of these possibilities.

- The number of $D S L$ receptors on neighboring cells, $N_{L}$. This parameter determines the steady-state value of $H$, and consequently, the steady state value of the maturation factor, $M$. Low value of $N_{L}$ will lead to low value of $H_{S}$ and high value of $M_{S}$. Therefore, the cell that receives from its neighbors low DSL signal will go into differentiation, independently, that is with no involvement of other environmental signals.

- The externally produced $D k k, D_{e}$. This value is added to the endogenous $D k k$. An increase in $D_{e}$ leads to an increase in the argument of the function $f_{W}^{\downarrow}$ and thus reduces this function values. Consequently, the r.h.s. of equation (3.9) decreases and $L_{S}$ decreases. The decrease in $L_{S}$ causes a direct decrease in $P_{S}$ and also in $N_{S}$ and $H_{S}$, the latter change increases $M_{S}$. Thus, it can be seen that $D_{e}$ serves as anti-proliferative and pro-maturation signal.

- The externally produced $W n t, W_{e}$. An increase in this parameter has an opposite effect to $D_{e}$ : it increases the values of $f_{W}^{\downarrow}$ and thus leads to an increase in $L_{S}$ and $P_{S}$ and to a decrease in $M_{S}$. Thus, it serves as an opposite signal to $D k k$, encouraging proliferation and blocking differentiation.

- The proportion of binding of cell E-cadherins by the E-cadherins of the adjacent cells, $k_{e}$. Increase in number of neighboring $E$-cadherins leads to an increase in the number of bound $E$-cadherins in steady state, $E_{S}$, and decreases $L_{S}$. The result is similar to the one described above, for $D_{e}$, namely, $k_{e}$ serves as anti-proliferative and pro-maturation signal.

\subsection{Interpreting the results - response to environmental conditions}

Let us biologically interpret the above analyzed mathematical properties of the system, in order to learn how the cell can integrate the micro-environmental signals into a full feedback control scheme. 
First, we study how the notion of stem cell niche can be represented by the molecular cell machinery and, specifically, by the Notch pathway. From our analysis we learn that the reduction of $D S L$ signalling, $N_{L}$, leads to an increase in $M_{S}$, independently of the level of $L$, that is, independently of the Wnt pathway. Moreover, it is biologically reasonable to assume that under low levels of all external signals the increase in $M$ is faster than the increase in $P$ due to the internal $W n t$ (i.e., $F_{W}^{\downarrow}$ being comparatively small for small $D_{e}$ and $W_{e}$ ). This means that for $N_{L}$ being small enough, $M$ will reach the threshold value and the cell will differentiate, prior to any change in its proliferative status. This suggests that the demand of minimal supporting niche is represented by this pathway.

If Notch signal is large enough, we can see that the balance between proliferation and differentiation is strongly influenced by the Wnt and $D k k$ signals, $W_{e}$ and $D_{e}$, respectively. At this point it is important to note that while Wnt is assumed to be produced by all the surrounding cells, $D k k$ is produced only by SCs. As a result, the signals depend both on the total number of neighbors and on the proportion of SCs in the micro-environment. The larger the number of neighbors, the larger is the proliferative signal via the Wnt pathway; the larger the number of SC neighbors, the larger is the anti-proliferative signal via the Dkk, thus supporting the previous Quorum Sensing assertion [2].

When the cell has a few neighbors, these supply external $W_{e}$, in addition to supplying $D S L$ receptors that prevent accumulating excessive $M$. The SCs among the neighbors also supply external $D_{e}$. Thus, increase in local density around the cell increases the levels of $L$ and $P$, while increase in the proportion of SCs in the neighborhood of the cell contributes to reduction in $L$ and $P$. The exact dynamics depend on the specific formulae for the functions used in the system. Clearly, however, these formulae can portray a threshold SC density, upon which the Dkk signal becomes dominant, elevates $M$, and consequently, leads to cell differentiation.

One important aspect of cell dynamics still needs to be discussed, namely, the proliferation arrest due to crowding of cells of any kind. In our system this role is played by the E-cadherin pathway and the external signal, represented by $k_{e}$. Increase in local cell density leads to high values of $k_{e}$, reducing $L_{S}$ and, consequently, $P_{S}$ and increasing $M_{S}$. Again, the exact properties depend on the form of the function $f_{L}^{\downarrow}$, but this function can be chosen so that for low values of $k_{e}$ its influence will be negligible, while for a larger argument it will reduce the value of the r.h.s. of (3.9) and therefore reduce $L_{S}$.

Now we can summarize this analysis, considering signal integration in different possible environments. In this summary we show how a proper choice of the explicit functions can lead to the experimentally observed SC behavior. Under extremely low local cell density, interpreted as being "outside the niche," low Notch signal leads to SC differentiation. When this is not the case, there is sufficient signal via the Notch pathway to prevent immediate differentiation. In such situations the intensities of $W n t, D k k$ and E-cadherin signals coming from neighboring cells determine cell fate, as detailed below.

- Under low cell density, $W_{e}$ is dominant on $k_{e}$ and $D_{e}$, and the SC is stimulated to proliferate; this can be achieved by choosing $f_{E}^{\downarrow}$ with relatively low sensitivity to its argument values, so 
that for constant $k_{e}$ there is relatively small change in the value of $f_{L}^{\downarrow}\left(k_{e} \cdot \frac{f_{E}^{\downarrow}\left(f_{W}^{\downarrow}\left(W_{e}, D_{e}+\frac{f_{D}^{\uparrow}(L)}{\mu_{D}}\right)\right)}{\mu_{E}}\right)$.

- Under intermediate local density and relatively low SC proportion, $W_{e}$ is still dominant over $D_{e}$ and SC proliferation takes place. In contrast, when proportion of SCs is relatively large, $D_{e}$ increases and reduces $L_{S}$. This can reduce $P_{S}$ below the threshold and arrest proliferation (if $f_{W}^{\downarrow}$ is sensitive enough to $D_{S}$ ).

- Under high local density, a large E-cadherin signal further reduces $f_{L}^{\downarrow}$ and $L_{S}$, causing a further decrease of $P_{S}$ and increase of $M_{S}$, which pushes the $M$ value above its threshold, so that the cell goes into differentiation.

We can conclude that by appropriate choice of specific form of the functions, the described control system can interpret the outside signals and drive the cell towards the desired response.

\section{Discussion}

Regulation of SC is an important domain of interest in biology and medicine. Due to the serious experimental and ethical challenges in SC research, a good understanding of SC behavior requires the use of mathematical methods for the interpretation of the empirical observations. Currently, extensive molecular research is conducted, which focuses on details of intracellular pathways, while other studies examine population properties. A mathematical effort is needed to establish the relations between these two scales of SC behavior. Using simple mathematical models we have outlined an approach to defining and exploring the interaction between intracellular and population scale.

In the present work we have analyzed a mathematical model for the intracellular pathways in human mammary SCs and used this analysis to examine how these pathways can serve as a control system that regulates cell fate and integrates signals from its environment. The main outcome of our work is the understanding that stem cell niche can be formed spontaneously in the tissue, rather than requiring external delimiting structures, as is currently thought. Moreover, the previous assertion that homeostasis in developing tissues is mainly controlled by a negative feedback on SC proliferation, denoted Quorum Sensing, is substantiated by this analysis, singling out the Dkk signal in the Wnt pathway as the Quorum Sensing "counter." Our analysis further suggests the relative dominance of the involved biochemical pathways in different tissue conditions.

A thorough experimental work is warranted for further characterizing the suggested control mechanism in the given cell population. Once verified experimentally, the model can be used to guide the laboratory research, by putting forward particular hypotheses and defining the format in which the measurements should be presented. For example, experiments could be devised to measure the responses of intracellular protein networks to well-defined environmental stimuli (addition of Wnt or other ligands), as well as the responses to different intracellular concentrations of the involved agents. Upon accumulation of experimental data, updates of the model can be introduced, 
e.g., further detailing of some of the pathways, which are now represented in a simplified way for facilitating the basic analysis.

Our analysis is not exhaustive. Global stability analysis can shed more light on this interesting system. Still, we believe that the present work is a first step in the future collaboration between theory and experiment to decipher the control mechanism of SC in developing tissues.

\section{Acknowledgments}

This work was supported by Chai Foundation and by Ministry of Immigrant Absorption of the State of Israel and by contract \# 012930 from EU FP6. The authors express their gratitude to Dr. Elina Azenshtein and Gennadiy Wasserman for useful discussion.

\section{References}

[1] Z. Agur, Y. Daniel, Y. Ginosar. The universal properties of stem cells as pinpointed by a simple discrete model. J. Math. Biol., 44 (2002), 79-86.

[2] Z. Agur, O.U. Kirnasovsky, L. Levi. Normal and pathological renewal of damaged tissues by stem cell homing analyzed in a simple discrete model. Submitted.

[3] B.D. Angst, C. MarcozziA.I. Magee. The cadherin superfamily: diversity in form and function. J. Cell Sci., 114 (2006), 629-641.

[4] D.W.H. Barnes, C.E. Ford, S.M. Gray, J.F. Louit. Spontaneous and induced changes in cell populations in heavily irradiated mice. Nuclear Energy Ser. VI, 2 (1959), 1-10.

[5] M. Baron. An overview of the Notch signalling pathway. Semin. Cell Dev. Biol., 14 (2003), 113-119.

[6] B. Barrilleaux, D.G. Phinney, D. Prockop, K.C. O'Connor. Review: ex vivo engineering of living tissues with adult stem cells. Tissue Eng., 12 (2006), No. 11, 3007-3019.

[7] J. Behrens, U. Frixen, J. Schipper, M. Weidner, W. Birchmeier. Cell adhesion in invasion and metastasis. Semin. Cell Biol., 3 (1992), 169-178.

[8] G. Berx, F. Nollet, F. van Roy. Dysregulation of the E-cadherin/catenin complex by irreversible mutations in human carcinomas. Cell Adhes. Commun., 6 (1998), 171-184.

[9] K.R. Brennan, A.M. Brown. Wnt proteins in mammary development and cancer. J. Mammary Gland Biol. Neoplasia, 9 (2004), 119-131.

[10] G. Dontu, W.M. Abdallah, J.M. Foley, K.W. Jackson, M.F. Clarke, M.J. Kawamura, M.S. Wicha. In vitro propagation and transcriptional profiling of human mammary stem/progenitor cells. Genes. Dev., 17 (2003), 1253-1270. 
[11] R.P. Hill, R. Perris. “Destemming” cancer stem cells. J. Natl. Cancer Inst., 99 (2007), No. $19,1435-40$.

[12] O.U. Kirnasovsky, Y. Kogan, Z. Agur. Resilience in stem cell renewal: development of the Agur-Daniel-Ginosar model. Submitted.

[13] O.U. Kirnasovsky, Y. Kogan, V. Vainstein, Z. Agur. Investigation of tumour dynamics by mathematical modelling at the cellular level. In preparation.

[14] O.U. Kirnasovsky, G. Wasserman, E. Azenshtein, Z. Agur. The molecular pathways controlling fate decision in mammary stem cells studied by a mathematical model. In preparation.

[15] E.C. Lai. Notch signaling: control of cell communication and cell fate. Development, 131 (2004), 965-973.

[16] M.T. Lewis, J.M. Veltmaat. Next stop, the twilight zone: hedgehog network regulation of mammary gland development. J. Mammary Gland Biol. Neoplasia, 9 (2004), 165-181.

[17] C.Y. Logan, R. Nusse. The Wht signaling pathway in development and disease. Annu. Rev. Cell Dev. Biol., 20 (2004), 781-810.

[18] W.E. Lowry, L. Richter. Signaling in adult stem cells. Front Biosci., 12 (2007), 3911-3927.

[19] M. Mimeault, R. Hauke, S.K. Batra. Stem cells: a revolution in therapeutics - recent advances in stem cell biology and their therapeutic applications in regenerative medicine and cancer therapies. Clin. Pharmacol. Ther., 82 (2007), No. 3, 252-264.

[20] A. O’Neill, D.V. Schaffer. The biology and engineering of stem-cell control. Biotechnol. Appl. Biochem., 40 (2004), 5-16.

[21] C.S. Potten, M. Loeffler. Stem cells: attributes, cycles, spirals, pitfalls and uncertainties. Lessons for and from the crypt. Development, 110 (1990), 1001-1020.

[22] T. Reya, H. Clevers. Wnt signalling in stem cells and cancer. Nature, 434 (2005), 843-850.

[23] F.W. Ruscetti, S. Akel, S.H. Bartelmez. Autocrine transforming growth factor-beta regulation of hematopoiesis: many outcomes that depend on the context. Oncogene, 24 (2005), 57515763.

[24] W. Shi, A.L. Harris. Notch signaling in breast cancer and tumor angiogenesis: cross-talk and therapeutic potentials. J. Mammary Gland Biol. Neoplasia, 11 (2006), 41-52. 